\title{
Concatenated Composite Pulses Compensating Simultaneous Systematic Errors
}

\author{
Masamitsu Bando $^{1 *}$, Tsubasa Ichikawa ${ }^{1 \dagger}$, Yasushi Kondo ${ }^{1,2}$ and Mikio Nakahara ${ }^{1,2}$ \\ ${ }^{1}$ Research Center for Quantum Computing, Interdisciplinary Graduate School of Science \\ and Engineering, Kinki University, 3-4-1 Kowakae, Higashi-Osaka, Osaka 577-8502, Japan \\ ${ }^{2}$ Department of Physics, Kinki University, 3-4-1 Kowakae, Higashi-Osaka, Osaka \\ 577-8502, Japan
}

In NMR experiments and quantum computation, many pulse (quantum gate) sequences called the composite pulses, were developed to suppress one of two dominant errors; a pulse length error and an off-resonance error. We describe, in this paper, a general prescription to design a single-qubit concatenated composite pulse (CCCP) that is robust against two types of errors simultaneously. To this end, we introduce a new property, which is satisfied by some composite pulses and is sufficient to obtain a CCCP. Then we introduce a general method to design CCCPs with shorter execution time and less number of pulses.

KEYWORDS: composite pulses, quantum gates, NMR

\section{Introduction}

Composite pulses $^{1-3)}$ are important techniques in nuclear magnetic resonance (NMR) experiments to suppress systematic errors in simple pulses such as a square pulse, which will be called "elementary pulses" in the following. Recently, they receive much attention particularly from a viewpoint of quantum computing. ${ }^{4,5)}$ Radio frequency pulses (rf-pulses) in NMR implement quantum gates, and composite pulses are employed to obtain high precision quantum gates that are robust against systematic errors. ${ }^{6-9)}$

In NMR, there are two typical errors, so called a pulse length error (PLE) and an offresonance error (ORE). ${ }^{3)}$ So far, most composite pulses were designed to fight against one of these two errors; BB1, ${ }^{10)}$ CORPSE and SCROFULOUS, ${ }^{11)}$ SK1, ${ }^{12,13)}$ and so on. Recently, two new composite pulses were introduced. One was due to Alway and Jones, ${ }^{14)}$ while the other was called the Knill pulse. ${ }^{15)}$ They are robust against these two types of errors simul-

\footnotetext{
${ }^{\dagger}$ Present address: Department of Physics, Gakushuin University, 1-5-1 Mejiro, Toshima-ku, Tokyo 171-8588, Japan

*E-mail: bando@alice.math.kindai.ac.jp
} 
taneously, although they are restricted within null operation and $\pi$-rotation (NOT gate) of a nuclear spin. In order to realize a composite pulse which is robust against these two errors simultaneously and without any restriction, we designed a ConCatenated Composite Pulse (CCCP) by concatenating CORPSE and SCROFULOUS. ${ }^{16)}$

The CCCP reported previously ${ }^{16)}$ consists of 3 composite pulses, each of which consists of 3 elementary pulses. Consequently, this CCCP is made of 9 pulses in total. Although this CCCP is robust against both PLE and ORE, its execution time is considerably longer than the corresponding elementary pulse. CCCPs made of less number of elementary pulses are certainly desirable from a viewpoint of decoherence suppression.

Establishing general prescription to design a CCCP and its improvement are the subjects of this paper. Some composite pulses have interesting property, which we call the residualerror-preserving (REP) property. Employing two types of composite pulses with mutually exclusive REP properties is essential to design a successful CCCP robust against both PLE and ORE. By using this method, we obtain many CCCPs systematically. Moreover, we further improve the method to reduce operation time and the number of elementary pulses of a CCCP.

In $\S 2$ and $\S 3$, we introduce the basics of pulses and introduce four well known composite pulses, BB1, CORPSE, SCROFULOUS and SK1. These composite pulses are robust against either PLE or ORE. In $\S 4$, we introduce the REP property to characterize composite pulses under two types of errors. Subsequently, we classify composite pulses according to the REP property, which is an important ingredient to design CCCPs. Then we describe the method to design successful CCCPs in $\S 5$. In $\S 6$, we show that the number of elementary pulses to form a CCCP can be reduced if the constituent composite pulse has identity elementary pulses as its components. We work out three examples of "reduced" CCCPs. The operation times and performances of CCCPs and reduced CCCPs are compared in $\$ 7$. Section 8 concludes this paper.

\section{Pulses}

Consider a two-level system (qubit). A pure state is represented by a point on the Bloch sphere and an operation of a single-qubit gate results in a rotation of the point around an axis through the centre of the Bloch sphere.

In NMR, we implement any rotations whose axes are in the $x y$-plane by controlling timings, strengths and durations of rf-pulses. ${ }^{17)}$ A single-qubit gate without an error in NMR quantum computation takes the form

$$
R(\theta, \phi)=\exp [-\mathrm{i} \theta \boldsymbol{n}(\phi) \cdot \boldsymbol{\sigma} / 2],
$$


where $\theta$ represents the rotation angle, $\phi$ is the azimuthal angle which specifies the rotation axis in the $x y$-plane as $\boldsymbol{n}(\phi)=(\cos \phi, \sin \phi, 0)$ and $\sigma=\left(\sigma_{x}, \sigma_{y}, \sigma_{z}\right)$ are the Pauli matrices. We call $R(\theta, \phi)$ of the form (1) an elementary pulse, following the NMR community convention.

In actual situation, however, errors are unavoidable and $R(\theta, \phi)$ is perturbed as

$$
R^{\prime}(\theta, \phi)=R(\theta, \phi)+O(\mathcal{E})
$$

where $\mathcal{E}$ specifies the strength of the error. Let $U(\theta, \phi)$ denote a sequence of $N$-pulses as

$$
U(\theta, \phi)=R\left(\theta_{N}, \phi_{N}\right) R\left(\theta_{N-1}, \phi_{N-1}\right) \cdots R\left(\theta_{1}, \phi_{1}\right),
$$

where the set of elementary pulses $\left\{R\left(\theta_{i}, \phi_{i}\right)\right\}$ is designed in such a way that $U(\theta, \phi)$ reproduces the desired error-free gate $R(\theta, \phi)$ as faithful as possible. Needless to say, $N$ should be taken as small as possible to avoid decoherence. Here we assume that the error strengths in all the elementary pulses are the same, which we will denote by $\mathcal{E}$ as before. Then eq. (3) is written as

$$
U^{\prime}(\theta, \phi)=R(\theta, \phi)-\mathrm{i} \mathcal{E} \delta+O\left(\mathcal{E}^{2}\right)
$$

where $U^{\prime}(\theta, \phi)$ represents $U(\theta, \phi)$ in the presence of error and $\delta U$ gives the structure of the first order error term. The set of elementary gates $\left\{R\left(\theta_{i}, \phi_{i}\right)\right\}$ is called a composite pulse if it is arranged to make $\delta U$ vanish so that

$$
U^{\prime}(\theta, \phi)=R(\theta, \phi)+O\left(\mathcal{E}^{2}\right) .
$$

\section{Composite Pulses in NMR}

In this section, we review typical composite pulses that are robust against one of two systematic errors in NMR: one is a PLE and the other is an ORE. For simplicity we henceforth ignore the second and higher order error terms.

\subsection{Pulse length error}

When a PLE is present, an elementary pulse $R(\theta, \phi)$ turns to

$$
R_{\varepsilon}^{\prime}(\theta, \phi)=R((1+\varepsilon) \theta, \phi) \approx R(\theta, \phi)-\mathrm{i} \varepsilon \theta(\boldsymbol{n}(\phi) \cdot \boldsymbol{\sigma}) R(\theta, \phi) / 2,
$$

where $R_{\varepsilon}^{\prime}(\theta, \phi)$ is the actual pulse in the presence of the PLE and $\varepsilon$ is an unknown but a fixed constant that represents the strength of the PLE. Some composite pulses robust against PLE are well-known in the NMR community: BB1, ${ }^{10)}$ SCROFULOUS ${ }^{11)}$ and SK1, ${ }^{12)}$ to name a few. The BB1 consists of four elementary pulses with parameters

$$
\theta_{1}=\theta_{3}=\pi, \quad \theta_{2}=2 \pi, \quad \theta_{4}=\theta,
$$




$$
\phi_{1}=\phi_{3}=\phi+\arccos [-\theta /(4 \pi)], \quad \phi_{2}=3 \phi_{1}-2 \phi, \quad \phi_{4}=\phi,
$$

while the SCROFULOUS consists of three elementary pulses with parameters

$$
\begin{array}{r}
\theta_{1}=\theta_{3}=\operatorname{arcsinc}[2 \cos (\theta / 2) / \pi], \quad \theta_{2}=\pi, \\
\phi_{1}=\phi_{3}=\arccos \left[-\pi \cos \theta_{1} /\left(2 \theta_{1} \sin (\theta / 2)\right)\right], \\
\phi_{2}=\phi_{1}-\arccos \left[-\pi /\left(2 \theta_{1}\right)\right],
\end{array}
$$

where $\operatorname{sinc} \theta=\sin \theta / \theta$. The SK1 also consists of three elementary pulses with parameters

$$
\begin{array}{r}
\theta_{1}=\theta, \quad \theta_{2}=\theta_{3}=2 \pi, \\
\phi_{1}=\phi, \quad \phi_{2}=\phi-\arccos [-\theta /(4 \pi)], \quad \phi_{3}=\phi+\arccos [-\theta /(4 \pi)] .
\end{array}
$$

To diminish the effects of random noises, geometric quantum gates ${ }^{18-20)}$ based on the holonomy ${ }^{21-24)}$ associated with the geometrical setting of the system have been proposed. All composite pulses that are robust against PLE are found to be geometric quantum gates. ${ }^{25,26)}$

\subsection{Off-resonance error}

When an ORE is present, an elementary pulse $R(\theta, \phi)$ turns to

$$
R_{f}^{\prime}(\theta, \phi)=\exp \left[-\mathrm{i} \theta\left(\boldsymbol{n}(\phi) \cdot \boldsymbol{\sigma}+f \sigma_{z}\right) / 2\right] \approx R(\theta, \phi)-\mathrm{i} f \sin (\theta / 2) \sigma_{z},
$$

where $R_{f}^{\prime}(\theta, \phi)$ is the actual elementary pulse in the presence of the ORE and $f$ is an unknown constant that characterizes the strength of the ORE. The CORPSE pulse sequence is the best known composite pulse to suppress ORE. ${ }^{11)}$ CORPSE consists of three elementary pulses

$$
\begin{array}{r}
\theta_{1}=2 n_{1} \pi+\theta / 2-k, \quad \theta_{2}=2 n_{2} \pi-2 k, \quad \theta_{3}=2 n_{3} \pi+\theta / 2-k, \\
\phi_{1}=\phi_{2}-\pi=\phi_{3}=\phi,
\end{array}
$$

where $k=\arcsin [\sin (\theta / 2) / 2]$ and $n_{i} \in \mathbb{Z}(i=1,2,3)$. It is common to take $n_{1}=n_{2}=1$ and $n_{3}=0$ for CORPSE and the one with $n_{1}=n_{3}=0, n_{2}=1$ is called the short CORPSE.

\subsection{Triviality of $N=2$ composite pulses}

We first define a pulse is trivial, regardress whether it is elementary or composite, when the resulting operation is the identity operation up to the overall phase. We now prove that there are no non-trivial $N=2$ composite pulses. We need at least $N=3$ in order to implement non-trivial composite pulses in a robust way. (It was shown in ${ }^{27)}$ that this is also the case for a two-qubit composite pulse robust against a $J$-coupling error.) 
Consider a composite pulse with $N=2$

$$
U(\theta, \phi)=R\left(\theta_{2}, \phi_{2}\right) R\left(\theta_{1}, \phi_{1}\right)
$$

First, suppose that this $U(\theta, \phi)$ is robust against a PLE. Equation (12) in the presence of PLE is written as

$$
U^{\prime}(\theta, \phi) \approx R(\theta, \phi)-\mathrm{i} \varepsilon R\left(\theta_{2}, \phi_{2}\right)\left[\left(\theta_{1} \boldsymbol{n}\left(\phi_{1}\right)+\theta_{2} \boldsymbol{n}\left(\phi_{2}\right)\right) \cdot \boldsymbol{\sigma}\right] R\left(\theta_{1}, \phi_{1}\right) / 2,
$$

where $R(\theta, \phi)=R\left(\theta_{2}, \phi_{2}\right) R\left(\theta_{1}, \phi_{1}\right)$. The robustness condition requires that the first order error term must vanish, which leads to

$$
\theta_{1} \boldsymbol{n}\left(\phi_{1}\right) \cdot \boldsymbol{\sigma}=-\theta_{2} \boldsymbol{n}\left(\phi_{2}\right) \cdot \boldsymbol{\sigma}
$$

This condition is satisfied if we make the following choice:

$$
\theta_{2}=-\theta_{1}, \quad \phi_{2}=\phi_{1} \quad \text { or } \quad \theta_{2}=\theta_{1}, \quad \phi_{2}=\phi_{1}+\pi
$$

Substituting eq. (15) to eq. (12), we find $U(\theta, \phi)=I$, where $I$ is the $2 \times 2$ identity operator. This proves triviality of any $N=2$ composite pulse robust against PLE.

Next, suppose that the composite pulse (12) is robust against the ORE. A composite pulse under ORE is written as

$$
U^{\prime}(\theta, \phi)=R(\theta, \phi)-\mathrm{i} f\left(\sin \left(\theta_{1} / 2\right) R\left(\theta_{2}, \phi_{2}\right)+\sin \left(\theta_{2} / 2\right) R^{\dagger}\left(\theta_{1}, \phi_{1}\right)\right) \sigma_{z} .
$$

Then the robustness condition is found to be

$$
\sin \left(\theta_{1} / 2\right) R(\theta, \phi)+\sin \left(\theta_{2} / 2\right) I=0
$$

which is satisfied if $\theta=2 \pi n(n \in \mathbb{Z})$. This proves that an $N=2$ composite pulse robust against ORE is trivial.

These two observations reveal that a non-trivial composite pulse robust against either PLE or ORE requires three elementary pulses or more.

\section{Residual-Error-Preserving Properties}

In this section, we introduce an important property of composite pulses that we call the Residual-Error-Preserving (REP) property. Consider a case in which both PLE and ORE are present. Then an elementary pulse is perturbed as

$$
R^{\prime}(\theta, \phi)=\exp \left[-\mathrm{i}(1+\varepsilon) \theta\left(\boldsymbol{n}(\phi) \cdot \boldsymbol{\sigma}+f \sigma_{z}\right) / 2\right] .
$$


Let us introduce $\mathcal{R}\left(\theta, \phi, \delta_{\varepsilon} U, \delta_{f} U\right)$, which is an elementary pulse taking into account two errors to the first order as

$$
\mathcal{R}\left(\theta, \phi, \delta_{\varepsilon} U, \delta_{f} U\right)=R(\theta, \phi)-\mathrm{i} \varepsilon \delta_{\varepsilon} U(\boldsymbol{n}(\phi) \cdot \boldsymbol{\sigma}) R(\theta, \phi) / 2-\mathrm{i} f \delta_{f} U \sigma_{z},
$$

where $\delta U_{\varepsilon}$ and $\delta U_{f}$ are matrices, in general, characterizing the first order error terms for PLE and ORE, respectively. According to this notation, the elementary pulse (18) is rewritten, to the first order, as

$$
R^{\prime}(\theta, \phi)=\mathcal{R}(\theta, \phi, \theta, \sin (\theta / 2))
$$

Note that both $\delta_{\varepsilon} U$ and $\delta_{f} U$ reduce to scalars $\theta$ and $\sin \theta / 2$, respectively, for an elementary gate.

Similarly, CORPSE is written in terms of $\mathcal{R}$ as

$$
\begin{aligned}
U_{\text {CORPSE }}^{\prime}(\theta, \phi) & =R^{\prime}\left(\theta_{3}, \phi\right) R^{\prime}\left(\theta_{2}, \bar{\phi}\right) R^{\prime}\left(\theta_{1}, \phi\right) \\
& =R(\theta, \phi)-\mathrm{i} \varepsilon\left(\theta_{1}-\theta_{2}+\theta_{3}\right) \boldsymbol{n}(\phi) \cdot \boldsymbol{\sigma} R(\theta, \phi) / 2 \\
& =R(\theta, \phi)-\mathrm{i} \varepsilon \theta \boldsymbol{n}(\phi) \cdot \boldsymbol{\sigma} R(\theta, \phi) / 2 \\
& =\mathcal{R}(\theta, \phi, \theta, 0),
\end{aligned}
$$

where the defining relations $\bar{\phi}=\phi+\pi$ and $\theta_{1}-\theta_{2}+\theta_{3}=\theta$ of CORPSE have been used. The last line of the above equation shows that $U_{\text {CORPSE }}^{\prime}(\theta, \phi)$ is regarded as the target elementary pulse under the influence of the PLE only (See eq. (6)), eliminating the effect of ORE. Similarly, $U_{\mathrm{SK} 1}^{\prime}(\theta, \phi)$ and $U_{\mathrm{BB} 1}^{\prime}(\theta, \phi)$, the SK1 and BB1 pulses, respectively, in the presence of two errors reduce to

$$
U_{\mathrm{SK} 1}^{\prime}(\theta, \phi)=U_{\mathrm{BB} 1}^{\prime}(\theta, \phi) \approx \mathcal{R}(\theta, \phi, 0, \sin (\theta / 2)),
$$

namely a pulse with the ORE only. In contrast, $U_{\mathrm{SCROF}}^{\prime}(\theta, \phi)$, the SCROFULOUS pulse to the first order, reduces to

$$
U_{\mathrm{SCROF}}^{\prime}(\theta, \phi)=\mathcal{R}\left(\theta, \phi, 0, \delta_{f} U\right)
$$

where

$$
\delta_{f} U=\left[\sin \theta_{1} \cot \left(\theta_{2} / 2\right)-2 \sin ^{2}\left(\theta_{1} / 2\right)\left(\cos \left(\phi_{1}-\phi_{2}\right)\right)+1\right] \sin \left(\theta_{2} / 2\right),
$$

which is different from $\delta_{f} U=\sin (\theta / 2)$ of an elementary pulse. SCROFULOUS behaves differently from an elementary pulse in this respect. This apparently minor difference plays an essential role in designing a composite pulse robust against two types of errors simultaneously. 
Table I. Residual-error-preserving (REP) property and robustness of composite pulses. The entry "REP" shows the type of REP property while the entry "robustness" shows the error type against which the composite pulse is robust. SK1, BB1 and CORPSE can be employed as an inner composite pulse, to be defined in the next section, in CCCP since they are REP.

\begin{tabular}{lcc}
\hline composite pulse & REP & robustness \\
\hline \hline SK1 & ORE & PLE \\
BB1 & ORE & PLE \\
SCROFULOUS & - & PLE \\
CORPSE $\left(n_{1}=n_{2}=1, n_{3}=0\right)$ & PLE & ORE \\
short CORPSE $\left(n_{1}=n_{3}=0, n_{2}=1\right)$ & - & ORE \\
\hline
\end{tabular}

It is found from eqs. (20), (21) and (22) that the first order PLE error term $\theta$ of CORPSE is the same as that of the target elementary pulse and CORPSE is regarded as an elementary pulse under PLE only, while the first order ORE error terms $\sin \theta / 2$ of SK1 and BB 1 are the same as those of the target elementary pulses and SK1 and BB1 are regarded as elementary pulses under ORE only. We call these properties "Residual-Error-Preserving" (REP).

With these observations, we introduce the following definitions.

- A composite pulse $U(\theta, \phi)$ robust against PLE satisfying the property

$$
U^{\prime}(\theta, \phi)=\mathcal{R}(\theta, \phi, 0, \sin (\theta / 2))
$$

is called residual-error-preserving with respect to ORE (REP-ORE).

- A composite pulse $U(\theta, \phi)$ robust against ORE satisfying the property

$$
U^{\prime}(\theta, \phi)=\mathcal{R}(\theta, \phi, \theta, 0)
$$

is called residual-error-preserving with respect to PLE (REP-PLE).

For example, CORPSE is REP-PLE, while SK1 and BB1 are REP-ORE. In contrast, SCROFULOUS does not have the REP property. Types of REP and robustness of well known composite pulses are summarized in Table I. The existence of REP property is important to design concatenated composite pulses as described in the next section.

\section{Concatenated Composite Pulses}

We now show how to design a concatenated composite pulse (CCCP). By concatenating two different composite pulses robust against two different types of errors, we obtain various CCCPs robust against both types of errors simultaneously. ${ }^{16)}$

Suppose there is a composite pulse robust against PLE (ORE). Now it should be clear 


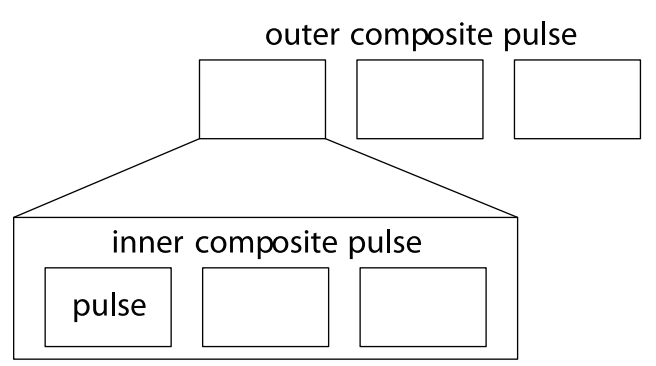

Fig. 1. Schematic diagram of outer composite pulse and inner composite pulse. Each elementary pulse in the outer composite pulse is replaced by the inner composite pulse. Time goes from left to right in this and the following pulse diagrams.

that we need to replace its constituent elementary pulses by other composite pulses which are robust against ORE (PLE) and REP-PLE (REP-ORE) to design a CCCP robust against PLE and ORE simultaneously. We call the former composite pulse outer while the latter one inner. Figure 1 explains this naming convention.

Let us confirm the above statement by explicitly examining the pulses. Let $V(\theta, \phi)=$ $R\left(\theta_{N}, \phi_{N}\right) \ldots R\left(\theta_{1}, \phi_{1}\right)$ be an outer composite pulse robust against PLE. Each elementary pulse $R\left(\theta_{i}, \phi_{i}\right)$ is replaced by an inner composite pulse $U\left(\theta_{i}, \phi_{i}\right)$ robust against ORE and are REP-PLE following the above prescription. The CCCP now takes the form $V(\theta, \phi)=$ $U\left(\theta_{N}, \phi_{N}\right) \ldots U\left(\theta_{1}, \phi_{1}\right)$. Under both PLE and ORE, the inner composite pulse is perturbed as $U^{\prime}\left(\theta_{i}, \phi_{i}\right)=\mathcal{R}\left(\theta_{i}, \phi_{i}, \theta_{i}, 0\right)$ by definition. This CCCP is robust against both types of errors since

$$
\begin{aligned}
V^{\prime}(\theta, \phi) & =U^{\prime}\left(\theta_{N}, \phi_{N}\right) \ldots U^{\prime}\left(\theta_{1}, \phi_{1}\right) \\
& =\mathcal{R}\left(\theta_{N}, \phi_{N}, \theta_{N}, 0\right) \ldots \mathcal{R}\left(\theta_{1}, \phi_{1}, \theta_{1}, 0\right) \\
& =\mathcal{R}(\theta, \phi, 0,0) .
\end{aligned}
$$

We have used the fact that $V$ is a composite pulse robust against PLE to derive the last equality.

Various CCCPs are obtained by choosing the outer and the inner composite pulses according to this scheme as listed in Table II. It was shown in $\$ 3$ that a non-trivial composite pulse robust against either the PLE or the ORE requires at least three pulses. Therefore the method to design a CCCP introduced here requires at least $N \geq 9$ pulses to implement a non-trivial CCCP.

\section{Reduced Concatenated Composite Pulses}

In this section, we employ composite pulses having trivial elementary pulses as constituents to reduce the number of elementary pulses in the resulting CCCP. Note that a trivial composite pulse robust against PLE or ORE can be constructed with only two elementary 
Table II. CCCPs obtained with our scheme and their inner and outer composite pulses. Note that the outer composite pulses of SKinsC and BBinsC are the short CORPSE.

\begin{tabular}{lcc}
\hline & \multicolumn{2}{c}{ composite pulse } \\
abbreviation of CCCP & inner & outer \\
\hline \hline CinS & CORPSE & SCROFULOUS \\
CinSK & CORPSE & SK1 \\
SKinsC & SK1 & short CORPSE \\
CinBB & CORPSE & BB1 \\
BBinsC & BB1 & short CORPSE \\
\hline
\end{tabular}

pulses and does not require three elementary pulses like in the case of non-trivial composite pulses as was proved in $\S 3$.

We use three trivial (composite) pulses to this end in the following. Let us first consider $R(\theta, \phi) R(\theta, \bar{\phi})$, which reduces to the identity operator when no errors are present. From eq. (6), the pulse sequence $R^{\prime}(\theta, \phi) R^{\prime}(\theta, \bar{\phi})$ under both PLE and ORE is

$$
\begin{aligned}
& R^{\prime}(\theta, \phi) R^{\prime}(\theta, \bar{\phi}) \\
& =R(0, \phi)-\mathrm{i} \varepsilon(\theta-\theta) \boldsymbol{n}(\phi) \cdot \boldsymbol{\sigma} R(0, \phi) / 2-2 \mathrm{i} f \sin (\theta / 2) R(\theta, \phi) \sigma_{z} \\
& =\mathcal{R}(0, \phi, 0,2 \sin (\theta / 2) R(\theta, \phi)),
\end{aligned}
$$

showing that the trivial pulse sequence $R(\theta, \phi) R(\theta, \bar{\phi})$ is robust against PLE.

An ideal pulse $R(2 \pi, \phi)$ and a pulse sequence $R\left(\pi, \phi^{\prime}\right) R(2 \pi, \phi) R\left(\pi, \phi^{\prime}\right)$ are trivial pulses up to the overall phase. These pulses under PLE and ORE are

$$
\begin{aligned}
R^{\prime}(2 \pi, \phi) & =-I+\mathrm{i} \varepsilon \pi \boldsymbol{n}(\phi) \cdot \boldsymbol{\sigma}-\mathrm{i} f \sin (\pi) \sigma_{z} \\
& =-\mathcal{R}(0, \phi, 2 \pi, 0),
\end{aligned}
$$

and

$$
\begin{aligned}
& R^{\prime}\left(\pi, \phi^{\prime}\right) R^{\prime}(2 \pi, \phi) R^{\prime}\left(\pi, \phi^{\prime}\right) \\
& =I-\mathrm{i} \varepsilon \pi\left(\boldsymbol{n}\left(\phi^{\prime}\right)+\boldsymbol{n}\left(2 \phi^{\prime}-\phi\right)\right) \cdot \boldsymbol{\sigma}+\mathrm{i} f\left[\left(\sigma_{z} R\left(\pi, \phi^{\prime}\right)+R\left(\pi, \phi^{\prime}\right) \sigma_{z}\right)\right] \\
& =\mathcal{R}\left(0, \phi, \delta_{\varepsilon} U, 0\right),
\end{aligned}
$$

where

$$
\delta_{\varepsilon} U=2 \pi\left[\exp \left(-\mathrm{i}\left(\phi^{\prime}-\phi\right) \sigma_{z}\right)+\exp \left(-2 \mathrm{i}\left(\phi^{\prime}-\phi\right) \sigma_{z}\right)\right]
$$

and we have used $\sigma_{z} R\left(\pi, \phi^{\prime}\right)=-R\left(\pi, \phi^{\prime}\right) \sigma_{z}$. Therefore these pulses are robust against the 
Table III. Robustness of three trivial composite pulses. Here $\bar{\phi}=\phi+\pi$. They are robust against either the PLE or the ORE.

\begin{tabular}{lc}
\hline pulse sequence & robustness \\
\hline \hline$R(\theta, \phi) R(\theta, \bar{\phi})$ & PLE \\
$R(2 \pi, \phi)$ & ORE \\
$R\left(\pi, \phi^{\prime}\right) R(2 \pi, \phi) R\left(\pi, \phi^{\prime}\right)$ & ORE \\
\hline
\end{tabular}

ORE. Table III summarizes robustness of these three trivial pulses. Although it might seem that the $N=3$ trivial composite pulse is useless, it has an important use in reduction of elementary pulses in a CCCP as we show in the following examples.

As far as we use concatenation, a composite pulse that is robust against both PLE and ORE must be composed of at least 5 pulses. As discussed in $\S 3$, the minimum number of components for the outer and inner composite pulses is three. And the outer composite pulse cannot be composed of trivial pulses only. Then we must have at least one non-trivial pulse as a constituent for the outer composite pulse (SK1 is an example). This pulse must be replaced by an inner composite pulse. For the trivial composite pulses that are robust against either PLE or ORE, there are options of $N=1,2$ and 3 as given in Table III. This proves that the outer composite pulse can contain one or more trivial composite pulses, and so the minimum $N$ for a CCCP is $1+1+3=5$. We show three convincing examples of reduced CCCPs below.

\subsection{Example 1}

The first example is the reduced CinSK. First, we replace a target pulse $R(\theta, \phi)$ by the SK1 (9). Next, we select the CORPSE as the inner composite pulse because it is robust against the ORE and REP-PLE. Since the SK1 is robust against PLE and has two trivial pulses $R(2 \pi, \phi \pm \arccos [-\theta /(4 \pi)])$ as the second and the third pulses, we need to replace only the first pulse by CORPSE, remembering that these trivial pulses are already robust against ORE. Therefore, we obtain a reduced CORPSE in SK1 CCCP (reduced CinSK) with $N=3+1+1=5$ pulses. The reduced CinSK is parameterized as

$$
\begin{array}{r}
\theta_{1}=\theta_{3}+2 \pi=2 \pi+\theta / 2-k, \quad \theta_{2}=2 \pi-2 k, \quad \theta_{4}=\theta_{5}=2 \pi, \\
\phi_{1}=\phi_{2}-\pi=\phi_{3}=\phi, \\
\phi_{4}=\phi-\arccos [-\theta /(4 \pi)], \quad \phi_{5}=\phi+\arccos [-\theta /(4 \pi)] .
\end{array}
$$

Schematic diagram to design the reduced CinSK is shown in Fig. 2. 


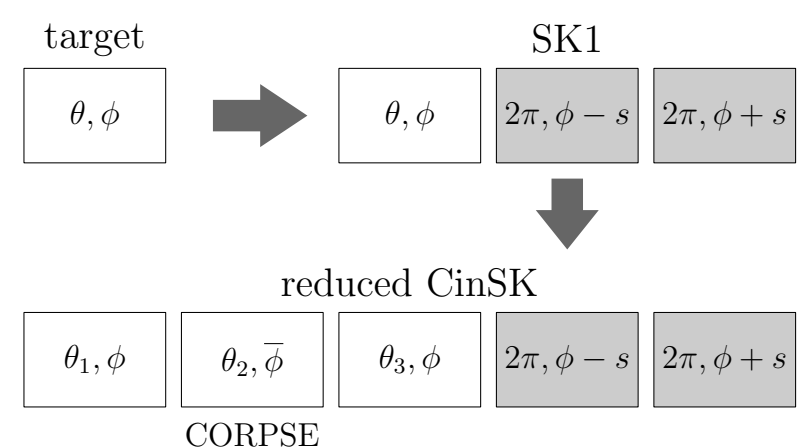

Fig. 2. Schematic diagram to design the reduced CinSK. Each box represents a pulse and is parameterized by the rotation angle and the azimuthal angle of the rotation axis. Here $s=\arccos [-\theta /(4 \pi)]$ and $\bar{\phi}=\phi+\pi$. Shaded boxes are pulses requiring no replacements by composite pulses.

\subsection{Example 2}

The second example is the reduced CinBB. First, we replace a target pulse $R(\theta, \phi)$ by BB1 (7), which is robust against PLE. Next, we select CORPSE as the inner composite pulse since it is robust against ORE and REP-PLE, which guarantees a successful CCCP. Since BB1 is robust against PLE and contains a trivial pulse sequence $R\left(\pi, \phi_{1}\right) R\left(2 \pi, \phi_{2}\right) R\left(\pi, \phi_{1}\right)$ that is robust against ORE, we need to replace only the fourth pulse in BB1 by CORPSE. As a result, we obtain the reduced CORPSE in BB1 CCCP (reduced CinBB) with $N=1+1+1+3=6$ elementary pulses. The reduced CinBB is parameterized as

$$
\begin{array}{r}
\theta_{1}=\theta_{3}=\pi, \quad \theta_{2}=2 \pi, \quad \theta_{4}=\theta_{6}+2 \pi=2 \pi+\theta / 2-k, \\
\theta_{5}=2 \pi-2 k, \quad \phi_{1}=\phi_{3}=\phi+\arccos [-\theta /(4 \pi)], \\
\phi_{2}=3 \phi_{1}-2 \phi, \quad \phi_{4}=\phi_{5}-\pi=\phi_{6}=\phi .
\end{array}
$$

Schematic diagram of the reduced CinBB is shown in Fig. 3.

\subsection{Example 3}

The last example is the reduced SKinsC. First, we replace a target pulse $R(\theta, \phi)$ by the short CORPSE robust against ORE. While the short CORPSE has no trivial pulse sequences, it is modified in order to include trivial pulse sequences. For this, here we use an important property of a pulse sequence $\prod_{i=1}^{N} R\left(\theta_{i}, \phi\right)$ with $\sum_{i=1}^{N} \theta_{i}=\theta$. This pulse sequence is equivalent to $R(\theta, \phi)$ in the error-free case, and from eq. (18), this pulse sequence under the PLE and the ORE becomes

$$
\prod_{i=1}^{N} R^{\prime}\left(\theta_{i}, \phi\right)=R^{\prime}(\theta, \phi) .
$$




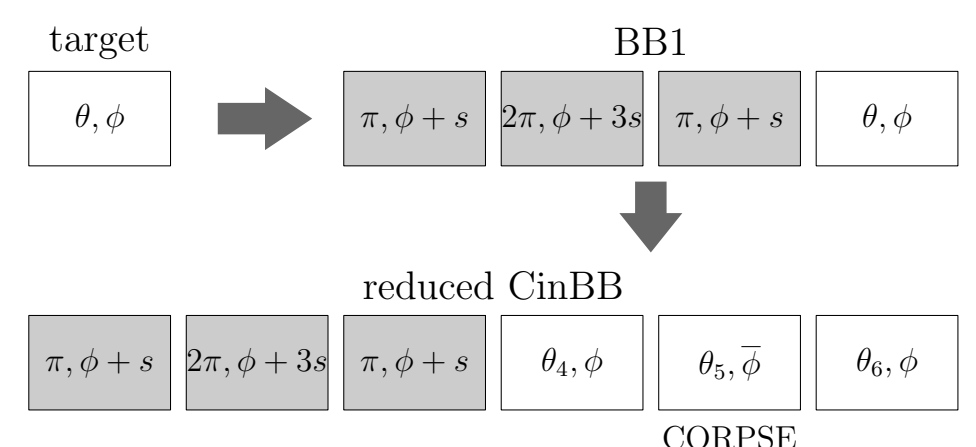

Fig. 3. Schematic diagrams to design the reduced CinBB. Each box represents a pulse and is parameterized by the rotation angles and azimuthal angle of the rotation axis, $s=\arccos [-\theta /(4 \pi)]$ and $\bar{\phi}=\phi+\pi$. Shaded boxes are pulses requiring no replacements by composite pulses.

Note that $R^{\prime}\left(\theta_{i}, \phi\right) R^{\prime}\left(\theta_{j}, \phi\right)=R^{\prime}\left(\theta_{i}+\theta_{j}, \phi\right)$ for $i, j=1,2, \ldots, N$ due to the commutativity $\left[R^{\prime}\left(\theta_{i}, \phi\right), R^{\prime}\left(\theta_{j}, \phi\right)\right]=0$. This shows the pulse sequence $\prod_{i=1}^{N} R\left(\theta_{i}, \phi\right)$ is both REP-PLE and REP-ORE. By using this result, we can modify the short CORPSE for the target elementary pulse $R(\theta, \phi)$ as follows:

$$
\begin{aligned}
& R\left(\theta_{3}, \phi\right) R\left(\theta_{2}, \bar{\phi}\right) R\left(\theta_{1}, \phi\right) \\
& \quad=\quad R\left(\theta_{3}, \phi\right) R\left(\theta_{3}, \bar{\phi}\right) R(2 \pi-\theta, \bar{\phi}) R\left(\theta_{1}, \bar{\phi}\right) R\left(\theta_{1}, \phi\right),
\end{aligned}
$$

where $\theta_{1}=\theta_{3}=\theta / 2-k, \theta_{2}=2 \pi-2 k$, and $k=\arcsin [\sin (\theta / 2) / 2]$. Two trivial pulse sequences $R\left(\theta_{1}, \bar{\phi}\right) R\left(\theta_{1}, \phi\right)$ and $R\left(\theta_{3}, \phi\right) R\left(\theta_{3}, \bar{\phi}\right)$ in the modified short CORPSE are robust against PLE as listed in Table III.

Next, we choose SK1 as an inner composite pulse since it is robust against PLE and REP-ORE. Since the modified short CORPSE has two trivial pulse sequences $R\left(\theta_{1}, \bar{\phi}\right) R\left(\theta_{1}, \phi\right)$ and $R\left(\theta_{3}, \phi\right) R\left(\theta_{3}, \bar{\phi}\right)$ robust against PLE, we need to replace only the third pulse by SK1. In addition, by using eq. (33), we can merge the second and the third pulses without affecting the robustness issue since $\phi_{2}=\phi_{3}$. Therefore, we obtain the reduced SK1 in modified short CORPSE CCCP (reduced SKinsC) which has $N=2+3+2-1=6$ pulses. The term -1 in $N$ accounts for the reduction of pulses by merging two pulses. The reduced SKinsC is parameterized as

$$
\begin{array}{r}
\theta_{1}=\theta_{5}=\theta_{6}=\theta / 2-k, \quad \theta_{2}=2 \pi-\theta / 2-k, \quad \theta_{3}=\theta_{4}=2 \pi, \\
\phi_{1}=\phi_{2}-\pi=\phi_{5}-\pi=\phi_{6}=\phi, \\
\phi_{3}=\phi-\arccos [-\theta /(4 \pi)], \quad \phi_{4}=\phi+\arccos [-\theta /(4 \pi)] .
\end{array}
$$

Schematic diagram to design the reduced SKinsC is shown in Fig. 4. 


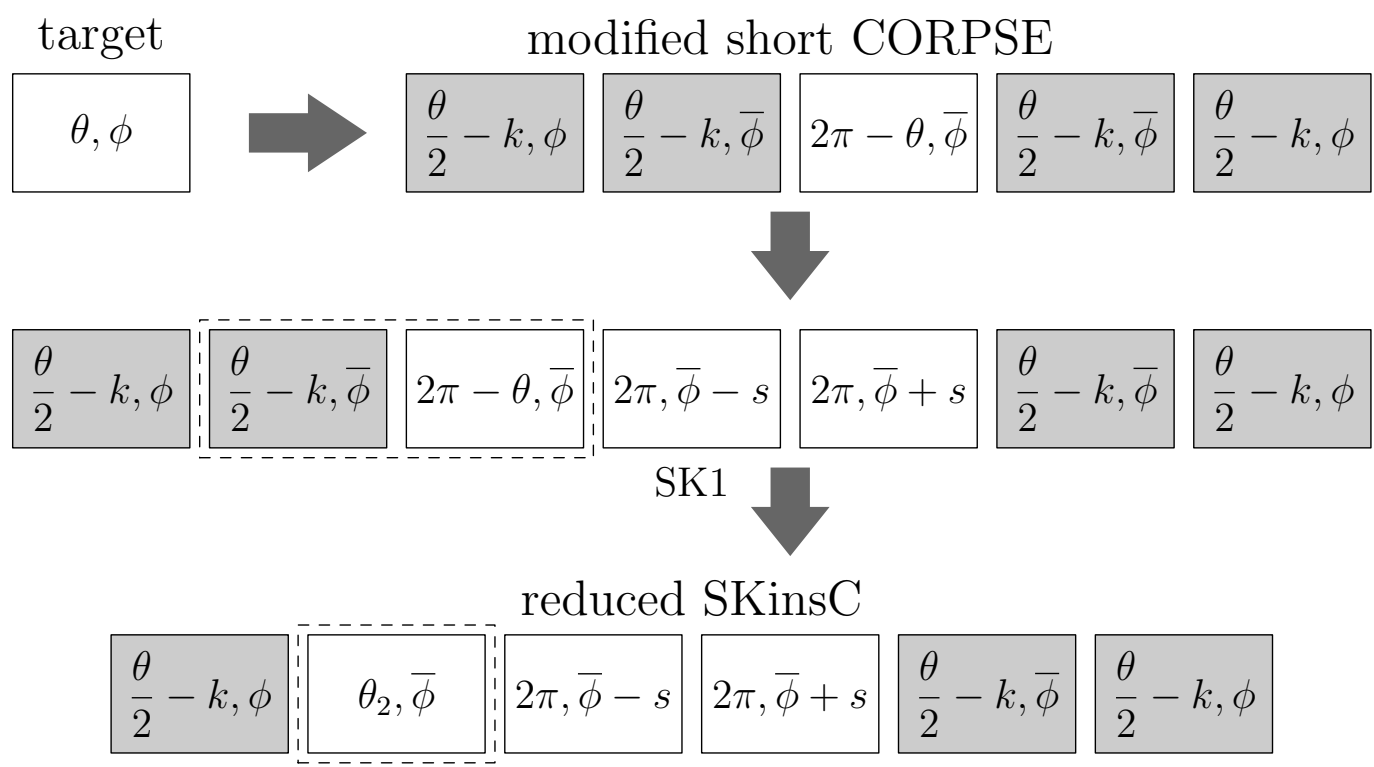

Fig. 4. Schematic diagram to design the reduced SKinsC. Each box represents a pulse and is parameterized by its rotation angle and the azimuthal angle of the rotation axis. Here $s=\arccos [-\theta /(4 \pi)], k=\arcsin [\sin (\theta / 2) / 2]$ and $\bar{\phi}=\phi+\pi$. Shaded boxes are pulses requiring no replacements by composite pulses. The second and the third pulses in the middle row are merged in the bottom row (surrounded by dashed boxes).

\section{Operation Time Cost and Fidelity}

A reduced CCCP has a shorter operation time than the original CCCP. In addition, generally, it has improved robustness thanks to the short operation time and less number of elementary pulses. We introduce two measures, the operation time cost $T$ and the gate (pulse) fidelity $F$, to compare performances of CCCPs.

The operation time cost $T$ is defined by

$$
T=\sum_{i=1}^{N} \theta_{i} / \pi
$$

where $\theta_{i}$ is a rotation angle of the $i$-th pulse.

As an example, let us consider CinSK parameterized as

$$
\begin{aligned}
& \theta_{1}=\theta_{3}+2 \pi=2 \pi+\theta / 2-k, \quad \theta_{2}=2 \pi-2 k, \\
& \theta_{4}=\theta_{7}=3 \pi, \quad \theta_{6}=\theta_{9}=\pi, \quad \theta_{5}=\theta_{8}=2 \pi .
\end{aligned}
$$

The operation time cost of CinSK is easily found as

$$
T=16+(\theta-4 k) / \pi .
$$


Table IV. Number of pulses, $N$, operation time cost $T$ and robustness of the elementary target pulse, composite pulses and CCCPs. $T(\pi / 2)$ and $T(\pi)$ are the operation time $\cos t T$ with the target rotation angle $\pi / 2$ and $\pi$, respectively. The "robustness" represents the error type against which the pulse is robust. Reduced CinBB and SKinsC attain approximately $50 \%$ reduction in the operation time cost compared to their nonreduced counterparts.

\begin{tabular}{lcccc}
\hline pulse & $N$ & $T(\pi / 2)$ & $T(\pi)$ & robustness \\
\hline \hline elementary & 1 & 0.5 & 1.0 & - \\
\hline SCROFULOUS & 3 & 2.3 & 3.0 & PLE \\
SK1 & 3 & 4.5 & 5.0 & PLE \\
BB1 & 4 & 4.5 & 5.0 & PLE \\
short CORPSE & 3 & 2.0 & 2.3 & ORE \\
CORPSE & 3 & 4.0 & 4.3 & ORE \\
\hline CinS & 9 & 12.5 & 13.0 & PLE, ORE \\
CinSK & 9 & 16.0 & 16.3 & PLE, ORE \\
CinBB & 12 & 18.7 & 19.0 & PLE, ORE \\
SKinsC & 9 & 14.0 & 14.3 & PLE, ORE \\
BBinsC & 12 & 14.0 & 14.3 & PLE, ORE \\
\hline reduced CinSK & 5 & 8.0 & 8.3 & PLE, ORE \\
reduced CinBB & 6 & 8.0 & 8.3 & PLE, ORE \\
reduced SKinsC & 6 & 6.0 & 6.3 & PLE, ORE \\
\hline
\end{tabular}

This should be compared with that of the reduced CinSK,

$$
T=8+(\theta-4 k) / \pi
$$

Number of elementary pulses, $N$, and the numerical values of operation time $\operatorname{cost} T$ for the elementary pulse, some composite pulses and CCCPs with the angle $\theta=\pi / 2$ and $\pi$ are listed in Table IV. It shows that a reduced CCCP achieves approximately 50\% reduction in the operation time cost compared to its nonreduced counterpart.

The gate fidelity of $U^{\prime}$ is defined by

$$
F=\left|\operatorname{tr}\left(U^{\dagger} U^{\prime}\right)\right| / 2
$$

where $U$ is an ideal pulse without errors corresponding $U^{\prime}$. The gate fidelity $F$ is a commonly used measure of a quantum gate accuracy based on the Hilbert-Schmidt inner product with respect to the one-qubit Hilbert space..$^{4,7,9,14)}$ The gate fidelity is a real number that takes a value $0 \leq F \leq 1$, and $F=1$ is achieved when there are no errors. Density plots of the gate fidelity of the target elementary pulse, BB1, CORPSE, and reduced CinBB are given in Fig. 5. These figures clearly show that the reduced CCCP is simultaneously robust against both PLE 

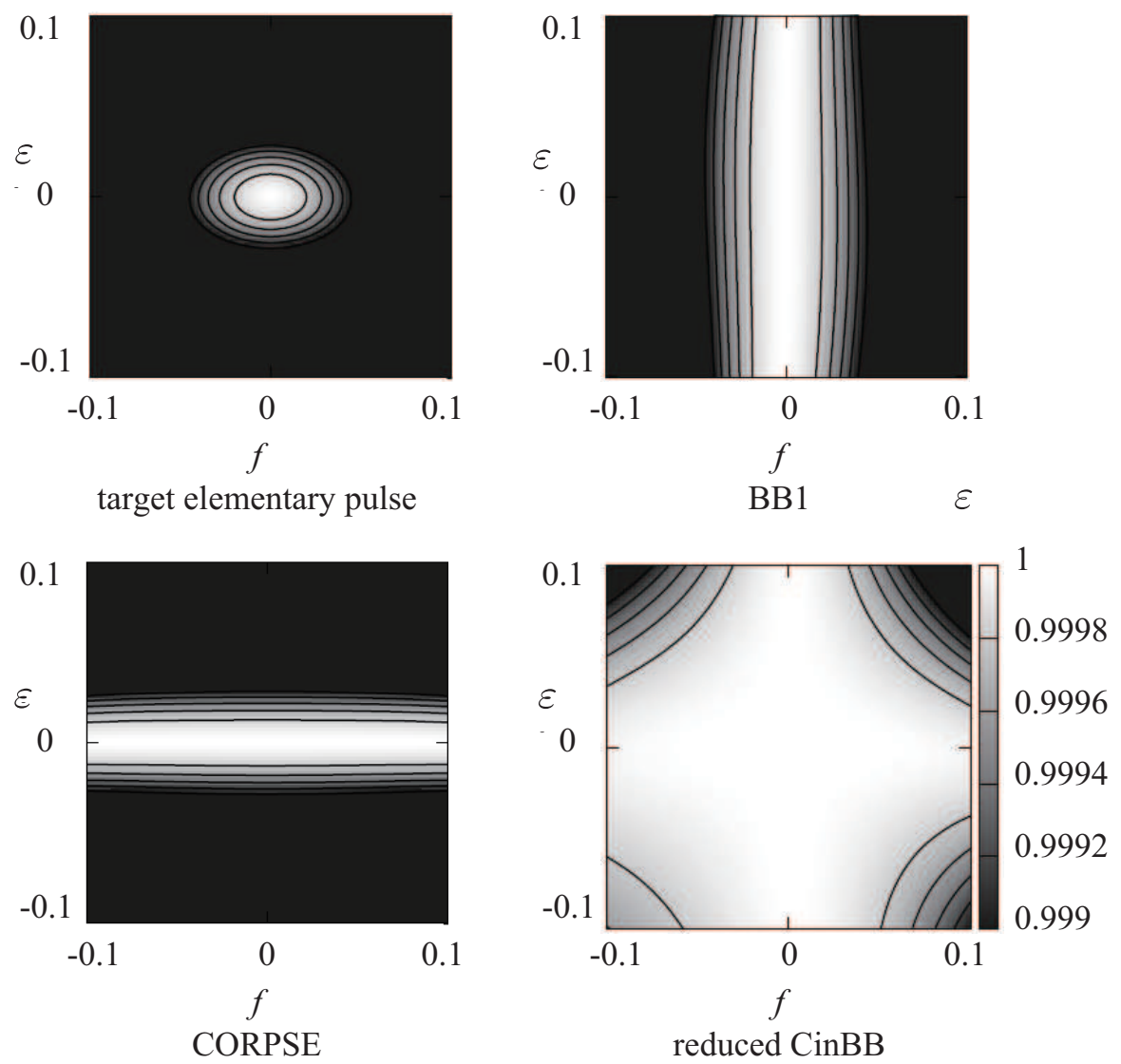

Fig. 5. Fidelity $F$ of a target elementary pulse, BB1, CORPSE, and reduced CinBB as a function of the error strengths $\varepsilon$ for PLE and $f$ for ORE. The target rotation angle is $\theta=\pi$. The reduced CinBB is robust against PLE and ORE simultaneously.

and ORE while others are not.

\section{Conclusion and Discussion}

Composite pulses proposed so far suppress either PLE or ORE. As a straightforward extension of conventional composite pulses, we establish a general method to design singlequbit concatenated composite pulses (CCCPs), which are robust against these two types of errors simultaneously. Some composite pulses have residual-error-preserving (REP) property that is a sufficient condition for them to be inner composite pulses of CCCPs. There are various combinations of two composite pulses to design CCCPs. If the composite pulses chosen have one or more trivial pulses, the number of pulses and operation time cost are further reduced.

In closing, we point out somewhat unexpected similarity between gate operations under error and those under noise. ${ }^{28)}$ For example, similar to our no-go theorem for $N=2$ composite pulse, it has been shown that dynamical decoupling pulses against any environment result 
in the identity operation. Moreover, a balanced pair, which is a two-pulse sequence whose first order noise term is identical to that of the elementary pulse, has been employed to implement a non-trivial pulse sequence robust against noise, which is similar to our residual-errorpreserving property. Whether this similarity is superficial or originates from deep connection between the two cases is under investigation and will be published elsewhere.

\section{Acknowledgment}

We would like to thank Lorenza Viola for drawing our attention to their work. ${ }^{28)}$ This work is supported by "Open Research Center" Project for Private Universities; Matching Fund Subsidy from MEXT (Ministry of Education, Culture, Sports, Science and Technology), Japan. M. B. is grateful to the Sasakawa Scientific Research Grant from The Japan Science Society. Y. K. and M. N. would like to thank partial supports of Grants-in-Aid for Scientific Research from the JSPS (Grant No. 23540470). 


\section{References}

1) C. Counsell, M. H. Levitt, and R. R. Ernst: J. Magn. Reson. 63 (1985) 133.

2) R. Tycko R, A, Pines, and J. Guckenheimer: J. Chem. Phys. 83 (1985) 2775.

3) M. H. Levitt: Prog. NMR Spectrosc. 18 (1986) 61.

4) M. A. Nielsen, and I. C. Chuang: Quantum Information and Quantum Computation (Cambridge University Press, Cambridge, 2000).

5) M. Nakahara, and T. Ohmi: Quantum Computing: From Linear Algebra to Physical Realizations (Taylor and Francis, Boca Raton, 2008).

6) J. A. Jones: Phil. Trans. R. Soc. Lond. A 361 (2003) 1429.

7) C. D. Hill: Phys. Rev. Lett. 98 (2007) 180501.

8) Y. Tomita, J. T. Merrill, and K. R. Brown: New J. Phys. 12 (2010) 015002.

9) J. A. Jones: Prog. NMR Spectrosc. 59 (2011) 91.

10) S. Wimperis: J. Magn. Reson. A 109 (1994) 221.

11) H. K. Cummins, G. Llewellyn, and J. A. Jones: Phys. Rev. A67 (2003) 042308.

12) K. R. Brown, A. W. Harrow, and I. L. Chuang: Phys. Rev. A70 (2004) 052318.

13) K. R. Brown, A. W. Harrow, and I. L. Chuang: Phys. Rev. A72 (2005) 039905(E).

14) W. G. Alway, and J. A. Jones: J. Magn. Reson. 189 (2007) 114.

15) C. A. Ryan, J. S. Hodges, and D. G. Cory: Phys. Rev. Lett. 105 (2010) 200402.

16) T. Ichikawa, M. Bando, Y. Kondo, and M. Nakahara: Phys. Rev. A84 (2011) 062311.

17) T. D. W. Claridge: High-Resolution NMR Techniques in Organic Chemistry (Elsevier, Oxford, 1999).

18) P. Zanardi, and M. Rasetti: Phys. Lett. A 264 (1999) 94.

19) S. -L. Zhu, and Z. D. Wang: Phys. Rev. Lett. 89 (2002) 097902.

20) Y. Ota, and Y. Kondo: Phys. Rev. A80 (2009) 024302.

21) M. V. Berry: Proc. R. Soc. Lond. A 392 (1984) 45.

22) F. Wilczek, and A. Zee: Phys. Rev. Lett. 52 (1984) 2111.

23) Y. Aharonov, and J. Anandan: Phys. Rev. Lett. 58 (1987) 1593.

24) M. Nakahara: Geometry, Topology and Physics, 2nd ed. (Taylor and Francis, Boca Raton, 2003). 
25) Y. Kondo, and M. Bando: J. Phys. Soc. Jpn. 80 (2011) 054002.

26) T. Ichikawa, M. Bando, Y. Kondo, and M. Nakahara: Phil. Trans. R. Soc. A 370 (2012) 4671.

27) T. Ichikawa, U. Güngördü, M. Bando, Y. Kondo, and M. Nakahara: arXiv:1204.2750.

28) K. Khodjasteh and L. Viola, Phys. Rev. A80 (2009) 032314. 